\title{
Schwinger mechanism during inflation
}

\author{
Soroush Shakeri, ${ }^{*}$ Mohammad Ali Gorji, ${ }^{\dagger}$ and Hassan Firouzjahi ${ }^{\ddagger}$ \\ School of Astronomy, Institute for Research in Fundamental Sciences (IPM), \\ P. O. Box 19395-5531, Tehran, Iran
}

(Received 16 March 2019; published 20 May 2019)

\begin{abstract}
We revisit the efficiency of the Schwinger mechanism in creating charged pairs during inflation. We consider a minimal setup of inflation in which the inflaton field is a complex scalar field charged under a $U(1)$ gauge field. There is a time-dependent conformal coupling which pumps energy from the inflaton field to the gauge field to furnish a nearly constant background electric field energy density to drive the Schwinger mechanism. The coupling between the gauge field and the scalar field induces a time-dependent effective mass for the inflaton field. The requirement of a long period of slow-roll inflation causes the Schwinger mechanism to be highly inefficient during inflation. The nonperturbative Schwinger mechanism can be relevant only toward the end of inflation and only on very small scales. This is in contrast to hypothetical models studied in literature in which the complex scalar field is a test field and a constant electric field is imposed on the dS background by hand. We calculate the number of pairs of charged particles created perturbatively during inflation. We show that it is proportional to the amplitude of the quadrupolar statistical anisotropy and it is very small. Consequently, the backreactions of created particles on magnetogenesis on large scales are negligible.
\end{abstract}

DOI: 10.1103/PhysRevD.99.103525

\section{INTRODUCTION}

The time dependent nature of the background, whether originating from gravitation or electric fields, leads to particle creation from vacuum [1]. In cosmology, the presence of a time-dependent background in an expanding Friedmann-Lemaître-Robertson-Walker universe leads to the spontaneous creation of particles out of the vacuum [2], while Hawking radiation is another example which is driven by the gravitational field in spacetimes with horizon such as black holes [3]. Moreover the existence of a strong enough electric field can create pairs of charged particles from vacuum by the Schwinger mechanism [4]. Therefore, the investigation of particle creation in the presence of both the gravitational and the electromagnetic (EM) fields is important for our understanding of the evolution of the universe, from the early time up to the late-time stages [5].

Pair production phenomenon with a background electric field in a de Sitter (dS) geometry has been extensively discussed using non-perturbative methods [6,7] (see also

\footnotetext{
s.shakeri@ipm.ir

'gorji@ipm.ir

firouz@ipm.ir
}

Published by the American Physical Society under the terms of the Creative Commons Attribution 4.0 International license. Further distribution of this work must maintain attribution to the author(s) and the published article's title, journal citation, and DOI. Funded by SCOAP ${ }^{3}$.
[8]), besides the perturbative ones [9]. More specifically, the Schwinger effect in Minkowski spacetime is a nonperturbative phenomenon which is exponentially suppressed below a critical electric field of about $1.3 \times 10^{18} \mathrm{~V} / \mathrm{m}$, so the direct evidence of this process has not been observed yet. This effect can be dynamically assisted by using additional EM fields revealing perturbative features of pair production [10]. While in the flat spacetime the perturbative computation of QED pair production has zero transition amplitude [11], but the perturbative pair production in $\mathrm{dS}$ in the presence of external EM fields is allowed.

The Schwinger effect in dS background has attracted much attentions in the past years. In the case of $1+1$-dimensional dS space, Schwinger effect revealed some quite different features thanks to the curvature of the spacetime $[6,12]$. It turns out that the current created by pair production increases as the electric field decreases which is different than our intuition of the Schwinger effect in the flat space. The extension of the setup to the case of $3+1 \mathrm{dS}$ background showed further unexpected results $[7,13,14]$. It is shown that the Schwinger effect has the similar aspects even in Ddimensional dS spacetime [15]. However, recently it is claimed that the unusual infrared (IR) behavior (negative conductivity) of the Schwinger current in dS background might be an artifact of regularization schemes [14]. The created pairs during the Schwinger process can backreact to the electromagnetic field in curved spacetime, causing some constraints on inflationary magnetogenesis models $[7,16,17]$. The created pairs move along the electric field and produce 
current and then change the conductivity of the ambient medium. The Schwinger effect is also explored as a reheating mechanism in the context of a relaxion model for inflation [18].

Apart from the backreaction of Schwinger pairs to the background EM fields, the time dependent nature of the background electric field in dS space has to be taken into account. While the constancy of the energy density in a homogeneous field configuration violates the second law of thermodynamics [19], most of the previous studies assume a constant electric field to consider Schwinger effect in dS space. In order to develop a more realistic model one may consider Schwinger pair production during an inflationary era. In this framework, besides the quasi-dS geometry which is implied by inflation, we need an electric field which is present during the inflationary period. In order to present such a setup we need to overcome several issues such as: (i) The existence of a background electric field and the associated induced charge current of the Schwinger process manifestly break the de Sitter invariance of the background geometry; (ii) Any vector field, like the electric field, is rapidly diluted because of the exponential expansion of the inflationary background. Providing the appropriate setup in order to tackle these problems is the main goal of the present paper.

The anisotropic inflation model provides a proper setup in which a persistent electric field can be maintained during inflation through a time-dependent gauge kinetic coupling, pumping energy continuously from the inflaton sector to the gauge field sector [20]. As a result, one has an attractor solution in which the energy density of the gauge field is a small and a nearly constant fraction of the total energy density which can last for a long enough period of inflation.

Recently several attempts were made in order to study Schwinger pair production by electric field coupled to inflaton and its backreaction to the background geometry $[17,21,22]$. This leads to some difficulties in solving the equations of motion of a charged scalar field to find the mode functions and interpret them in terms of positive and negative frequency modes [21]. Moreover, it is shown that the Schwinger effect during inflation will cause an angular dependence on the primordial power spectrum and bispectrum [23]. In fact the charged particle production rate depends on the direction of these particles with respect to the background electric field and therefore leaves a unique angular dependence on the primordial spectra.

In all of these studies the complex scalar field, which is responsible for the pair production, is considered to be a test field during inflation while the $\mathrm{dS}$ spacetime is driven by the real inflaton field. In other words, the Schwinger mechanism of pair creation is decoupled from the inflationary sector. In addition, in most of these models, no mechanism is provided to generate the background constant electric field to drive the Schwinger mechanism.
To address these shortcomings, in this work we present a minimal setup in which both the inflaton field and the charged scalar field are the same. We employ the charged extension of the anisotropic inflation [20] presented in $[24,25]$ where the inflaton is charged under a $U(1)$ gauge field. At the background level, the model is physically the same as the anisotropic inflation. In this model the inflaton field drives the quasi-de Sitter expansion through the slowroll conditions while simultaneously pumping energy into the gauge field sector to furnish a nearly constant background electric field. At the level of perturbations, the pairs are naturally produced from the quantum fluctuations of the complex inflaton field. We show that the conventional nonperturbative Schwinger effect is important only at the late stages of inflation. Since in most of the inflationary era the electric field energy density is required to be small to allow slow-roll inflation, pair production can only happen perturbatively. Note that the particle creation by a cosmological anisotropic Bianchi I universe in the presence of a constant electric field has been considered before in [26].

Since the quantum fluctuations of the inflaton field also generate the curvature perturbations [25], the parameters space of the model are tightly restricted by the CMB observations [27]. Moreover, the anisotropic inflation model predicts quadrupolar statistical anisotropies in the CMB angular power spectrum which are highly constrained by CMB observations [28]. Therefore some of the unusual aspects of the Schwinger mechanism in dS space which were obtained in previous studies (as summarized above) may be the results of a large and unrealistic parameter space in which no links between observations and theoretical results were made.

The rest of the paper is organized as follows. In Sec. II we review the model of charged anisotropic inflation and explain how a nearly constant electric field in quasi-de Sitter background arises in this model. In Sec. III the perturbation analysis of the model are presented. In Sec. IV we compute the charged pair production rate while in Sec. V the power spectra of the curvature and isocurvature modes are presented, followed by summaries and discussions in Sec. VI.

\section{THE MODEL}

In order to study the Schwinger process during inflation, we consider the model studied in [24], containing a charged complex scalar field as the inflaton field and a $U(1)$ gauge field $A_{\mu}$,

$$
\begin{aligned}
S= & \int d^{4} x \sqrt{-g}\left[\frac{M_{P}^{2}}{2} R-\frac{1}{2} D_{\mu} \varphi \overline{D^{\mu} \varphi}\right. \\
& \left.-\frac{1}{2} m^{2} \varphi \bar{\varphi}-\frac{1}{4} f^{2}(\varphi, \bar{\varphi}) F_{\mu \nu} F^{\mu \nu}\right],
\end{aligned}
$$

where the field strength tensor $F_{\mu \nu}=\partial_{\mu} A_{\nu}-\partial_{\nu} A_{\mu}$ and the covariant derivative is given by $D_{\mu}=\partial_{\mu}+i \mathbf{e} A_{\mu}$ in which $\mathbf{e}$ 
is the gauge coupling constant. This model is an extension of the anisotropic inflation model [20] to the case of complex inflaton field where its perturbation analysis were studied in [25,27]. The isotropic version of the model is also recently proposed in Ref. [29] where the inflaton is charged under a triplet of $U(1)$ gauge fields.

Here we briefly review the main results in this setup at the background level which are studied in detail in $[24,25,27]$.

In the model (1) the gauge kinetic coupling $f$ breaks the conformal invariance such that the background gauge field survives the exponential expansion. While this coupling represents a nonrenormalizable interaction, this kind of term is usually used for inflationary model buildings in low energy expansions in the spirit of the effective field theory. For the sake of simplicity, we assume an axially symmetric structure in field space and therefore the coupling function becomes only a function of the amplitude of the complex field $\varphi \bar{\varphi}=|\varphi|^{2}$ so $f(\varphi, \bar{\varphi})=f(|\varphi|)$. Taking this assumption into account, it is better to write the complex field in the polar coordinates as

$$
\varphi=\rho e^{i \theta}
$$

where $\rho$ represents the amplitude while $\theta$ is the phase. Working with the above polar coordinates makes the calculations simpler. The coupling function then is only a function of the radial coordinates, $f(|\varphi|)=f(\rho)$.

Varying the action (1) with respect to the gauge field $A_{\mu}$, we find the Maxwell equations

$$
\nabla_{\mu}\left(\sqrt{-g} f^{2} F^{\mu \nu}\right)=\mathbf{e} J^{\nu}
$$

where we have defined the 4-current as

$$
J^{\nu} \equiv \rho^{2} \sqrt{-g}\left(\partial^{\nu} \theta+\mathbf{e} A^{\nu}\right)
$$

which satisfies the continuity equation $\nabla_{\mu} J^{\mu}=0$.

The existence of a background gauge field clearly breaks the isotropy and we have to consider a Bianchi spacetime for the background geometry. However, when studying the cosmological perturbations, we can neglect the effects of the anisotropy in geometry as far as the size of anisotropy is sufficiently small. The statistical anisotropies in cosmological observables are predominantly induced from the matter (electric field) perturbations [25,27]. Therefore, we consider the isotropic Friedmann-Lemaitre-RobertsonWalker background geometry

$$
d s^{2}=-d t^{2}+a(t)^{2} \delta_{i j} d x^{i} d x^{j},
$$

where $a(t)$ is the scale factor. For the gauge field, we take the following time-dependent configuration

$$
A_{\mu}=(0, A(t), 0,0)
$$

which preserves homogeneity while breaking the isotropy.

Using Eqs. (2) and (6), the time component of the Maxwell equation (3) implies $\dot{\theta}=0$ which shows that the phase $\theta$ does not play any role at the level of background. This is the advantage of working with the polar variables $\rho$ and $\theta$ rather than the original fields $\varphi$ and $\bar{\varphi}$.

The spatial components of the Maxwell equations (3) yields

$$
\partial_{t}\left(f^{2} a \dot{A}\right)=-\mathbf{e}^{2} a \rho^{2} A .
$$

In comparison with the standard anisotropic inflation [20], the right-hand side of the Maxwell equation has the nonzero current coming from the induced mass term by the gauge field. This term also induces an effective mass $\mathbf{e}^{2} A_{\mu} A^{\mu}$ for the inflaton field. However, in order not to destroy the slow-roll condition, i.e., the inflaton mass to be small compared to the Hubble expansion rate during inflation, the induced mass term should be negligible during most of the period of inflation [25]. The background is therefore similar to the case of standard anisotropic inflation setup while interesting features of the models with the effects of $\mathbf{e}^{2}$ appear at the level of perturbations [27].

The $0-0$ component of the Einstein equations yields the Friedmann equation

$3 M_{P}^{2} H^{2}=\frac{1}{2} \dot{\rho}^{2}+\frac{1}{2} m^{2} \rho^{2}+\frac{1}{2} a^{-2} f^{2} \dot{A}^{2}+\frac{1}{2} \mathbf{e}^{2} a^{-2} \rho^{2} A^{2}$,

where $H(t)=\dot{a} / a$ is the Hubble parameter and a dot indicates the derivative with respect to the cosmic time. Combining the above result with the $i-i$ component of the Einstein equations, we find the time evolution of the Hubble parameter as

$$
M_{P}^{2} \dot{H}=-\frac{1}{2} \dot{\rho}^{2}-\frac{1}{3} a^{-2} f^{2} \dot{A}^{2}
$$

Since the charged mass term $\frac{1}{2} \mathbf{e}^{2} a^{-2} \rho^{2} A^{2}$ behaves as a potential term giving a mass to the inflaton field, it did not appear in the time evolution of the Hubble parameter.

The right-hand side of (8) is the total energy density where the first two terms are the standard energy density of the inflaton. The third term is the kinetic energy of the gauge field coming from the coupling between the inflaton and the Maxwell terms while the last term comes from the charged mass term which, as we discussed above, is only important at the final stages of inflation. As soon as the induced mass term by the gauge field $\left(\mathbf{e}^{2} A_{\mu} A^{\mu} \rho^{2}\right)$ dominates, it terminates inflation quickly.

Taking the variation with respect to $\varphi$, we obtain the Klein-Gordon equation as 


$$
\ddot{\rho}+3 H \dot{\rho}+m^{2} \rho=a^{-2} f f_{, \rho} \dot{A}^{2}-\mathbf{e}^{2} a^{-2} \rho A^{2},
$$

where $f_{, \rho}=\partial_{\rho} f$. The above equation shows the direct coupling between the inflaton and gauge field in this model.

Our model describes two different phases: (i) inflationary stage when the effects of the charged mass term are negligible and the setup is very similar to the standard anisotropic inflation model containing a real inflaton field [20]. (ii) When the charged mass term dominates, for instance the last term in Eq. (8), so it quickly terminates slow-roll inflation. During the first stage, which is most of the period of inflation, we can neglect the charged mass term in all the background equations (7), (8), and (10).

Now, we should choose an explicit functional form for the coupling function $f(\rho)$. In the case of coupling of axion with gauge fields, the desired symmetry of the system under consideration uniquely fixes the functional form so that the coupling function is linearly proportional to the axion (see Refs. [30]). Here, however, we deal with a phenomenological inflationary scenario and, as shown in [20], with an appropriate form of the conformal coupling $f(\rho)$, the gauge field drags energy continuously from the inflaton sector which prevents the dilution of the vector field in the exponentially expanding universe. This is an attractor solution in which the electric field energy density reaches a small but a nearly constant fraction of the total energy density. Let us elaborate more on this effect. During most of the period of inflation the effects of the induced mass term $\mathbf{e}^{2} A_{\mu} A^{\mu} \rho^{2}$ are negligible so we can easily integrate the Maxwell equation (7) obtaining

$$
\dot{A}=\frac{q_{0}}{a f^{2}},
$$

where $q_{0}$ is an integration constant.

Note that the functional form of the gauge coupling function $f(\rho)$ determines the time dependence of the electric field in the model. The third term in the righthand side of Friedmann equation (8) is the energy density of the vector field which in the case of $f=1$ decays like $a^{-4}$. In order to prevent this dilution, using Eq. (11), it can be seen that if we choose $f \propto a^{-2}$, the energy density of the vector field remains constant. This result at background level determines the functional form of $f(\rho)$ [20]

$$
f(\rho)=\exp \left(\frac{c \rho^{2}}{2 M_{P}^{2}}\right)
$$

where $c \geq 1$ is a parameter. With this form of $f(\rho)$, the system reaches the attractor regime in which the gauge field's energy density becomes a constant fraction of the total energy density [20].

Alternatively, the time-dependence of $f(\rho)$ can be written as

$$
f=\left(\frac{\tau}{\tau_{e}}\right)^{2 c}
$$

where $\tau$ is the conformal time defined as $\tau=\int d t / a(t)$ and $\tau_{e}$ denotes the time of the end of inflation.

Substituting the above result, the energy density of the vector field turns out to be

$$
\rho_{E} \equiv \frac{1}{2} a^{-2} f^{2} \dot{A}^{2}=\frac{1}{2} q_{0}^{2} H^{4} \tau_{e}^{4 c} \tau^{4(1-c)} .
$$

From the above relation we see that the energy density of the vector field is almost constant during the inflation. Now, it is easy to interpret the integration constant $q_{0}$. Demanding $\left.\rho_{E}\right|_{\tau=\tau_{e}}=E_{0}^{2} / 2$, we find

$$
q_{0}=\frac{E_{0}}{H^{2} \tau_{e}^{2}},
$$

where $E_{0}$ is the amplitude of the electric field at the end of inflation. Substituting Eqs. (13) and (15) in Eq. (11) and then integrating, we find that

$$
A(\tau)=\frac{1}{-4 c+1} \frac{E_{0}}{H^{2} \tau}\left(\frac{\tau_{e}}{\tau}\right)^{4 c-2} .
$$

During the final stages of inflation when $\tau \sim \tau_{e}$ we find $A(\tau) \approx-\frac{E_{0}}{H^{2} \tau}$ which is the same as the ansatz supposed in [7]. However, note the important difference that during most of period of inflation $A(\tau)$ scales like $A(\tau) \sim \tau^{-3}$ which is quite different from the ansatz employed in [7] and in other works dealing with setup similar to [7]. This is the key difference which significantly reduces the efficiency of the Schwinger mechanism during inflation.

During the attractor phase, the contribution of the gauge field to the total energy density of the model is given by the ratio [20]

$$
R \equiv \frac{\rho_{E}}{\rho_{\phi}}=\frac{E_{0}^{2} f^{-2} a^{-4}}{m^{2} \rho^{2}+\dot{\rho}^{2}}=\frac{c-1}{2 c} \epsilon=\frac{I}{2} \epsilon,
$$

where we have introduced the anisotropy parameter $I \equiv$ $(c-1) / c$ and $\epsilon$ is the slow-roll parameter which is given by

$$
\epsilon=-\frac{\dot{H}}{H^{2}}=\frac{1}{2 c}\left(\frac{V_{, \phi}}{V}\right)^{2} .
$$

It is worth mentioning that there are no limitations on the values of $c$ and $I$ at the background level except that $c \geq 1$. However, at the perturbation level, in order to satisfy the observational constraints on CMB anisotropies [28] one requires that $I \lesssim 10^{-7}[25,31,32]$.

To make the qualitative behavior of the model more transparent, in Fig. 1(a) we have plotted the phase space diagram of $(\rho, \dot{\rho})$ for the fixed value of $\mathbf{e}=0.01$ and for 


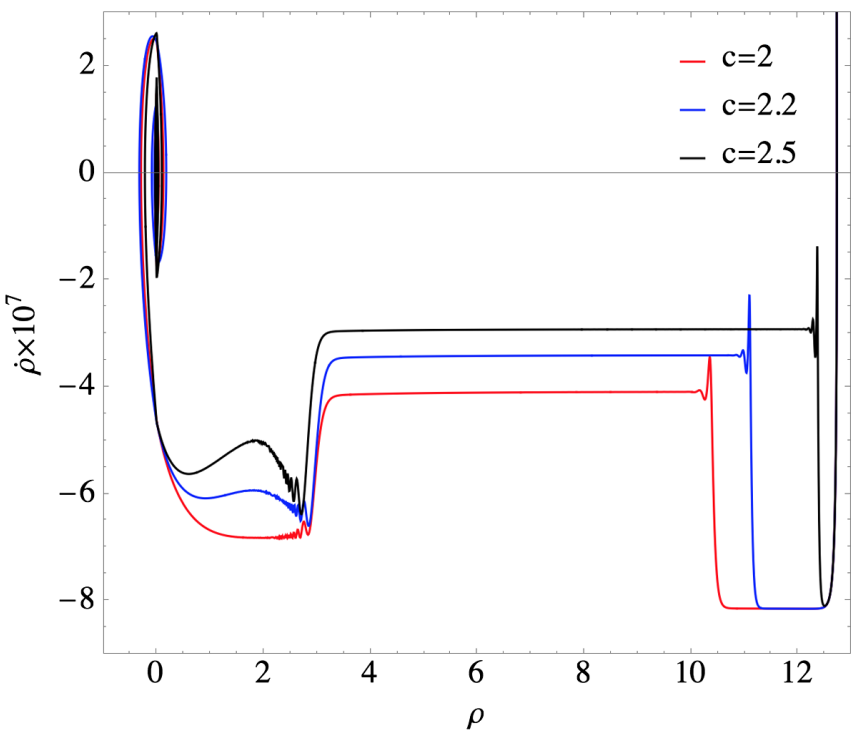

(a)

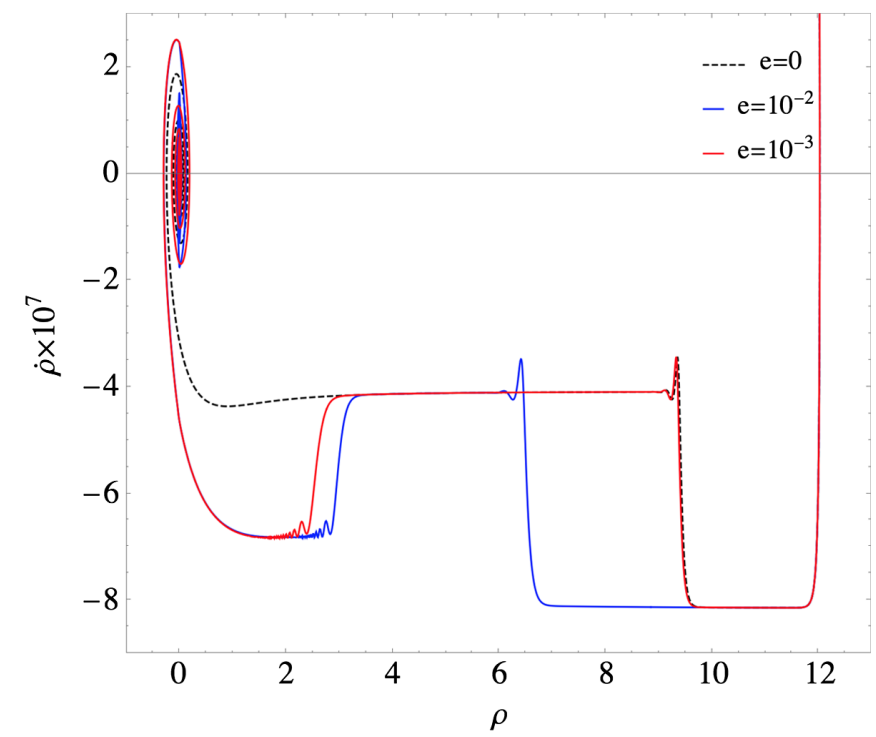

(b)

FIG. 1. (a) The phase space plot with $\mathbf{e}=0.01$ for different values of $c=2,2.2$ and 2.5 from top to bottom, respectively. We have also set $m=10^{-6} M_{P}, \rho(0)=12 M_{P}$ and $\dot{\rho}(0)=0$. (b) The phase space plot with $c=2$ for different values of $\mathbf{e}=0,10^{-2}$ and $10^{-3}$ from top to bottom, respectively. As in left plot, $m=10^{-6} M_{P}, \rho(0)=12 M_{P}$ and $\dot{\rho}(0)=0$.

different values of $c=2,2.2$ and 2.5. As it is clear from this figure the starting time of the attractor phase depends on the value of $c$ parameter. The larger values of $c$ correspond to pumping more energy into the gauge field and, consequently, the attractor phase happens earlier. In Fig. $1 \mathrm{~b}$, the phase space diagram of $(\rho, \dot{\rho})$ for the fixed value of $c=2$ but for different values of $\mathbf{e}=0,10^{-2}$ and $10^{-3}$ are plotted. As can be seen from this figure, the role of e becomes important only at the final stage of inflation. This is because the gauge field scales like $A \propto 1 / \tau^{3}$ [see Eq. (16)] so the induced mass term $\mathbf{e}^{2} A_{\mu} A^{\mu} \rho^{2}$ becomes important only toward the end of inflation when the inflaton field become massive, violating the slow-roll conditions.

\section{PERTURBATION ANALYSIS}

In this section, we present the cosmological perturbations of the model. For the purpose of Schwinger pair creation, we are interested only in the scalar perturbations.

The scalar perturbations around the background geometry (5) are given by

$$
\begin{aligned}
d s^{2}= & -a^{2}\left(1+2 N_{1}\right) d \tau^{2}+2 a B_{, i} d \tau d x^{i} \\
& +a^{2}\left((1+2 \psi) \delta_{i j}+E_{, i j}\right) d x^{i} d x^{j},
\end{aligned}
$$

where $N_{1}, B, \psi$, and $E$ are the scalar modes.

Scalar perturbations in the matter sector are given by

$$
\begin{aligned}
\varphi & \rightarrow \rho+\delta \varphi, \quad A_{\mu} \rightarrow A_{\mu}+\delta A_{\mu}, \quad \text { with } \\
\delta A_{\mu} & =\left(\delta A_{0}, \delta A_{x}, \partial_{y} M, 0\right),
\end{aligned}
$$

where $\delta \varphi, \delta A_{0}, \delta A_{x}$, and $M$ are the scalar modes in the matter sector.

In total, we have nine scalar modes. However, not all of these modes are physical degrees of freedom. The spacetime diffeomorphism invariance fixes two scalar modes. We choose to work in the spatially flat gauge with

$$
\psi=E=0 .
$$

Moreover, the $U(1)$ invariance of the matter sector fixes one of the scalar modes. We work in Coulomb gauge $\partial^{i} \delta A_{i}=0$, which implies

$$
\partial^{x} \delta A_{x}=-\partial^{y} \partial_{y} M
$$

From the above relation, one of the scalar modes can be solved in terms of the other. Fixing the gauges, then we have six scalar modes. The direct calculations show that the modes $N_{1}, B$, and $\delta A_{0}$ are nondynamical which can be solved from a set of algebraic equations of motion and then substituted back into the quadratic action of the remaining perturbations. As shown in $[25,27]$ these non-dynamical modes are slow-roll suppressed [27] and their contributions can be neglected to leading orders in slow-roll parameters. Note that the perturbation analysis for the gauge fields and the charged inflaton without neglecting the nondynamical constraint equations has been considered in [33].

Going to the Fourier space and using the twodimensional rotational symmetry in $y-z$ plane in the small anisotropy limit and considering $\mathbf{k}=\left(k_{x}, k_{y}, 0\right)=k(\cos \theta$, $\sin \theta, 0$ ), from the Coulomb gauge condition Eq. (22) we find $M=-i \frac{k_{x}}{k_{y}^{2}} \delta A_{x}$. After substituting this in Eq. (20), we obtain 


$$
\delta A_{\mu}=\delta A_{x}\left(0,1,-\frac{k_{x}}{k_{y}}, 0\right) .
$$

The gauge field excitations $\delta A_{\mu}$ can also be decomposed into the transverse mode $D_{1}$ and the longitudinal mode $D_{2}$, which are related to $\delta A_{x}$ and $M$ as

$$
\begin{gathered}
D_{1} \equiv \delta A_{x}-i k \cos \theta M, \\
D_{2} \equiv \cos \theta \delta A_{x}+i k \sin ^{2} \theta M .
\end{gathered}
$$

Using the Coulomb gauge (22), it is easy to show that

$$
D_{1}=\frac{\delta A_{x}}{\sin ^{2} \theta}, \quad D_{2}=0,
$$

which shows that the longitudinal mode does not propagate in the Coulomb gauge (22).

In summary, neglecting the contributions of the nondynamical modes, we have only three dynamical scalar modes $\left(\delta \varphi, \overline{\delta \varphi}, D_{1}\right)$ which propagate in a quasi-dS background.

Implementing the following field redefinitions for the canonically normalized fields $q$ and $D$,

$$
q_{k} \equiv a \delta \varphi_{k}, \quad D_{k} \equiv f \sin \theta D_{1 k},
$$

and after performing some integration by parts, the quadratic action to leading orders in terms of the small parameters $I$ and $\epsilon$ is obtained to be

$$
\begin{aligned}
S_{2}= & \frac{1}{2} \int d^{3} k d \tau\left[\left|q_{k}^{\prime}\right|^{2}-\left(k^{2}-\frac{2+6 I}{\tau^{2}}+\frac{3 \epsilon}{\tau^{4} H^{2}}+\frac{\mathbf{e}^{2} I \epsilon}{3 \tau^{2} H^{2}}\left(\frac{\tau_{e}}{\tau}\right)^{4}\right)\left|q_{k}\right|^{2}\right. \\
& +\frac{3 I}{\tau^{2}}\left(q_{k}^{2}+\bar{q}_{k}^{2}\right)+D_{k}^{\prime 2}-\left(k^{2}-\frac{2}{\tau^{2}}+\frac{2 \mathbf{e}^{2}}{\tau^{2} H^{2} \epsilon}\left(\frac{\tau_{e}}{\tau}\right)^{4}\right) D_{k}^{2} \\
& \left.-2 \sqrt{\frac{2 I}{3}} \frac{\sin \theta}{\tau^{2}}\left(6-\frac{\mathbf{e}^{2}}{H^{2}}\left(\frac{\tau_{e}}{\tau}\right)^{4}\right)\left(q_{k}+\bar{q}_{k}\right) D_{k}+2 \sqrt{6 I} \frac{\sin \theta}{\tau}\left(q_{k}+\bar{q}_{k}\right) D_{k}^{\prime}\right],
\end{aligned}
$$

where we have set $M_{P}=1$ and a prime denotes derivative with respect to the conformal time. To obtain the above action, we have used $\rho=\sqrt{\frac{2}{\epsilon}}$ and $A=\sqrt{\frac{I \epsilon}{3}} a \frac{\tau_{e}^{2}}{\tau^{2}}$ which are obtained from the background equations.

The complex scalar modes $\bar{q}_{k}$ and $q_{k}$ are responsible for the Schwinger pair production process in our formalism. The corresponding equation of motion for $q$ can be obtained from the action (28) as follows

$$
\begin{aligned}
q_{k}^{\prime \prime} & +\left(k^{2}-\frac{2}{\tau^{2}}+\frac{m^{2}}{H^{2} \tau^{2}}+\frac{\mathbf{e}^{2} E_{0}^{2}}{9 H^{4} \tau^{2}}\left(\frac{\tau_{e}}{\tau}\right)^{4}\right) q_{k} \\
& =-4 \sqrt{2} \frac{E_{0}}{\sqrt{\epsilon} H} \sin \theta\left(\left(1-\frac{\mathbf{e}^{2} \tau_{e}^{4}}{6 H^{2} \tau^{4}}\right) \frac{D_{k}}{\tau^{2}}-\frac{D_{k}^{\prime}}{2 \tau}\right) .
\end{aligned}
$$

It is instructive to compare Eq. (29) with Eq. (2.13) of Ref. [7]. The authors in Ref. [7] looked at a test complex scalar field in a dS background in the presence of a constant background electric field. Here, however, the quantum fluctuations of the inflaton field are responsible for the Schwinger pair production process. Moreover, in Ref. [7], a constant electric field in a dS spacetime is imposed by hand while in our model the electric field is driven by the dynamics of the model. Besides, in our inflationary setup, the slow-roll conditions provide a quasi-dS setup and the gauge kinetic coupling $f(\rho)$ prevents the electric field from being diluted during inflation. Here, a constant background electric field can be obtained via $f \propto a^{-2}$ (i.e., with $c \simeq 1$ ).
In this regard, our inflationary scenario is more natural with a minimum number of parameters to study the Schwinger process during an inflationary era.

Now let us elaborate more on the Mukhanov-Sasaki equation (29) to study the Schwinger process and charged particle production in our model. Our Eq. (29) is different than the corresponding equation used in previous works, e.g., Eq. (2.13) of [7] and those in [14,17,21-23] studying the Schwinger effect in cosmological scenarios, in two aspects: (i) In our case the quantum fluctuations of the electric field, encoded in the scalar mode $D_{k}$, source the quantum fluctuation of the complex field and therefore they indirectly contribute to the pair production process. (ii) The last term in the left-hand side of Eq. (29) is the effects of the background field in pair production process which is proportional to $a^{6}$ while it was proportional to $a^{2}$ in Ref. [7]. The later result is also included in Refs. [21,22] where a complex test field is considered in the context of anisotropic inflation [20]. Note that in our model, the quantum fluctuations of the inflaton field are responsible for the Schwinger process of generating charged pair particles.

In this paper, we have two types of charged pair production: those coming from the background electric field which are encoded in the last term in the left-hand side of Eq. (29) and those coming from the quantum fluctuations of the gauge field in the right-hand side of Eq. (29). The first is similar to what usually arise in nonperturbative analysis of the Schwinger effect (see for instance Eq. (2.13) of Ref. [7]) while the latter is a new type of perturbative 
source for the charged pair production. In previous studies, the last term in the left-hand side of Eq. (29) which was imposed by hand in the strong electric field regime, was responsible for the nonperturbative Schwinger mechanism in a dS background. However, in our analysis this term is small during most of the period of inflation. In other words we are working in the weak electric field regime.

Note that the last term in the left-hand side of Eq. (29) is proportional to $\tau^{-6}$ and it dominates toward the end of inflation $\tau \rightarrow 0$. So, there is a critical time $\tau_{c}$ beyond which the perturbative approximation breaks down and Eq. (29) is no longer applicable. Though we should consider the nonperturbative Schwinger effect after $\tau_{c}$ but the time range is very short and inflation ends quickly once we cross $\tau_{c}$. In order to find the critical time $\tau_{c}$, let us work with the real and imaginary parts of the complex field $q$ :

$$
q \equiv v+i u, \quad \bar{q} \equiv v-i u .
$$

In terms of the new fields defined in Eq. (30), the quadratic action (28) takes the following form

$$
\begin{aligned}
S_{2}= & \frac{1}{2} \int d^{3} k d \tau\left[u_{k}^{\prime 2}-\left(k^{2}-\frac{2+3 \epsilon}{\tau^{2}}\right.\right. \\
& \left.+\frac{3 \epsilon}{\tau^{4} H^{2}}+\frac{\mathbf{e}^{2} I \epsilon}{3 \tau^{2} H^{2}}\left(\frac{\tau_{e}}{\tau}\right)^{4}\right) u_{k}^{2} \\
& +v^{\prime 2}-\left(k^{2}-\frac{2+3 \epsilon+12 I}{\tau^{2}}+\frac{3 \epsilon}{\tau^{4} H^{2}}+\frac{\mathbf{e}^{2} I \epsilon}{3 \tau^{2} H^{2}}\left(\frac{\tau_{e}}{\tau}\right)^{4}\right) v_{k}^{2} \\
& +D_{k}^{\prime 2}-\left(k^{2}-\frac{2}{\tau^{2}}+\frac{2 \mathbf{e}^{2}}{\tau^{2} H^{2} \epsilon}\left(\frac{\tau_{e}}{\tau}\right)^{4}\right) D_{k}^{2} \\
& \left.-4 \sqrt{6 I} \sin \theta\left(\frac{2 D_{k}}{\tau^{2}}-\frac{D_{k}^{\prime}}{\tau}+\frac{\mathbf{e}^{2}}{3 H^{2}}\left(\frac{\tau_{e}}{\tau}\right)^{4} \frac{D_{k}}{\tau^{2}}\right) v_{k}\right] .
\end{aligned}
$$

One advantage of working with these new variables is that the quantum fluctuations of the electric field only couples with $v$ since $q+\bar{q}=2 v$ and the imaginary component $u$ decouples completely.

The equations of motion for the modes $v$ and $D$ can be obtained from the action (31) as follows

$$
\begin{aligned}
v_{k}^{\prime \prime} & +\left(k^{2}-\frac{2+12 I}{\tau^{2}}+\frac{3 \epsilon}{H^{2} \tau^{4}}+\frac{I \epsilon \mathbf{e}^{2} M_{P}^{2}}{3 H^{2} \tau^{2}}\left(\frac{\tau_{e}}{\tau}\right)^{4}\right) v_{k} \\
& =2 \sqrt{6 I} \sin \theta\left(\frac{2 D_{k}}{\tau^{2}}-\frac{D_{k}^{\prime}}{\tau}-\frac{\mathbf{e}^{2} M_{P}^{2}}{3 H^{2}}\left(\frac{\tau_{e}}{\tau}\right)^{4} \frac{D_{k}}{\tau^{2}}\right),
\end{aligned}
$$

and

$$
\begin{aligned}
D_{k}^{\prime \prime} & +\left(k^{2}-\frac{2}{\tau^{2}}+\frac{2 \mathbf{e}^{2} M_{P}^{2}}{\epsilon H^{2} \tau^{2}}\left(\frac{\tau_{e}}{\tau}\right)^{4}\right) D_{k} \\
& =2 \sqrt{6 I} \sin \theta\left(\frac{v_{k}}{\tau^{2}}+\frac{v_{k}^{\prime}}{\tau}-\frac{\mathbf{e}^{2} M_{P}^{2}}{3 H^{2}}\left(\frac{\tau_{e}}{\tau}\right)^{4} \frac{v_{k}}{\tau^{2}}\right) .
\end{aligned}
$$

We will estimate the critical time $\tau_{c}$ as the time in which the system of coupled equations (32) and (33) cannot be treated perturbatively. In order to do this, we should compare the interaction terms with each other in both equations. Let us first look at the mode $D$ in Eq. (33). There are two types of interaction terms. The self-interaction term containing $\frac{2 \mathbf{e}^{2} M_{P}^{2}}{\epsilon H^{2} \tau^{2}}\left(\frac{\tau_{e}}{\tau}\right)^{4}$ in the left-hand side of Eq. (33) and the interaction with the mode $v$ in the right-hand side of Eq. (33) giving rise to $2 \sqrt{6 I} \sin \theta \frac{\mathrm{e}^{2} M_{P}^{2}}{3 H^{2} \tau^{2}}\left(\frac{\tau_{e}}{\tau}\right)^{4}$. Comparing these terms with each other, and noting that $\sqrt{\frac{2 I}{3}} \epsilon \ll 1$, we find that the self-interaction term dominants earlier. Therefore, the critical time at which the mode $D$ starts to show nonperturbative behavior is determined by the selfinteraction term, given by

$$
\tau_{D}=\tau_{e}\left(\frac{\mathbf{e}^{2} M_{P}^{2}}{\epsilon H^{2}}\right)^{\frac{1}{4}}=\tau_{e}\left(\frac{\mathbf{e}^{2}}{8 \pi^{2} \epsilon^{2} \mathcal{P}_{R}}\right)^{\frac{1}{4}},
$$

where $\mathcal{P}_{R}=\frac{H^{2}}{8 \pi^{2} M_{P}^{2} \epsilon} \sim 2.1 \times 10^{-9}$ is the curvature perturbation power spectrum.

In the same manner, for the mode $v$ we should compare the two types of interactions in Eq. (32) such as $\frac{I \epsilon \mathbf{e}^{2} M_{P}^{2}}{3 H^{2} \tau^{2}}\left(\frac{\tau_{e}}{\tau}\right)^{4}$ and $2 \sqrt{6 I} \sin \theta \frac{\mathrm{e}^{2} M_{P}^{2}}{3 H^{2}}\left(\frac{\tau_{e}}{\tau}\right)^{4}$. The ratio of these interaction terms is $\sqrt{\frac{8}{3}} \frac{\sin \theta}{\sqrt{I} \epsilon}$. Therefore we define the critical time associated to these interactions as

$$
\tau_{v}=\tau_{e}\left(\frac{I \mathbf{e}^{2}}{48 \pi^{2} \mathcal{P}_{R}}\right)^{\frac{1}{4}}
$$

From Eqs. (35) and (34), we find

$$
\tau_{v}=\tau_{D}\left(\frac{I \epsilon^{2}}{6}\right)^{\frac{1}{4}}
$$

As discussed before, the anisotropic inflationary models generate statistical anisotropies which are tightly constrained by the $\mathrm{CMB}$ data [28]. In order not to generate large statistical anisotropy we require $I \lesssim 10^{-7}$ $[24,25,31,32]$. In addition, in order for the tensor perturbations to be perturbatively under control, we require $\mathbf{e} \lesssim$ $10^{-3}$ [27]. Taking the slow-roll parameter to be $\epsilon \sim 10^{-2}$, we find (note that during inflation $\tau<0$ )

$$
\tau_{D}<\tau_{e} \lesssim \tau_{v}
$$

This is an interesting result indicating that we can study the mode $v$ perturbatively from the past infinity to the end of inflation $\left(-\infty, \tau_{e}\right]$, while the mode $D$ becomes nonperturbative near the end of inflation. On the other hand, the mode $v$ is responsible for the charged pair production. This analysis shows that the nonperturbative Schwinger pair production does not take place during most of period of inflation and may be relevant only towards the end of 
inflation. This conclusion is the key difference of our model compared to other less realistic scenarios studied in previous works on Schwinger mechanism during inflation. This is because we took the complex scalar field to be the inflaton field itself which is responsible for curvature perturbations, and not a hypothetical test field decoupled from inflation.

\section{PAIR PRODUCTION}

In this section, we quantize the fields and find the number of charged pairs which are produced perturbatively during inflation.

From the action (28) we obtain the conjugate momenta for the charged quantum fluctuations as $\Pi_{q}=\bar{q}^{\prime}$ and $\bar{\Pi}_{q}=q^{\prime}$. Then promoting $q$ to quantum operator and expanding them in terms of the mode functions, we have

$$
\hat{q}(\tau, \mathbf{x})=\int \frac{d^{3} k}{(2 \pi)^{3}}\left(a_{-\mathbf{k}} q_{k}(\tau)+b_{\mathbf{k}}^{\dagger} \bar{q}_{-k}(\tau)\right) e^{-i \mathbf{k} . \mathbf{x}},
$$

in which the mode function $q_{k}$ satisfies Eq. (29) accordingly.

Demanding the commutation relations $\left[\hat{q}(\tau, \mathbf{x}), \hat{\Pi}\left(\tau, \mathbf{x}^{\prime}\right)\right]=$ $i \delta^{(3)}\left(\mathbf{x}-\mathbf{x}^{\prime}\right)$ while all other commutation relations being zero, we find the following well-known commutation relations between the annihilation and creations operators

$$
\left[a_{\mathbf{k}}, a_{\mathbf{k}^{\prime}}^{\dagger}\right]=\left[b_{\mathbf{k}}, b_{\mathbf{k}^{\prime}}^{\dagger}\right]=(2 \pi)^{3} \delta^{(3)}\left(\mathbf{k}-\mathbf{k}^{\prime}\right) .
$$

In the same manner, we quantize the quantum fluctuation of gauge field as

$$
\hat{D}(\tau, \mathbf{x})=\int \frac{d^{3} k}{(2 \pi)^{3}}\left(c_{-\mathbf{k}} D_{k}(\tau)+c_{\mathbf{k}}^{\dagger} \bar{D}_{k}(\tau)\right) e^{-i \mathbf{k} . \mathbf{x}},
$$

with

$$
\left[c_{\mathbf{k}}, c_{\mathbf{k}^{\prime}}^{\dagger}\right]=(2 \pi)^{3} \delta^{(3)}\left(\mathbf{k}-\mathbf{k}^{\prime}\right)
$$

in which the mode function $D_{k}$ satisfies Eq. (33).

The correlation functions (power spectra) for the free parts of the modes $v, u$, and $D$ can be simply obtained if we neglect the effects of gauge field in background dynamics by setting $I=0$. More precisely, we can solve the mode functions $v(\tau), u(\tau)$ and $D(\tau)$ for the limit $I=0$. Then we take into account the effects of the gauge field on the inflaton field and the pair production perturbatively in terms of small parameters $I$ and $\mathbf{e}$.

Before going further it is useful to express $u$ and $v$ in terms of the annihilation and creation operators. Substituting Eq. (38) in definition (30), we find

$$
\begin{aligned}
\hat{v}(\tau, \mathbf{x})= & \int \frac{d^{3} k}{(2 \pi)^{3}} \hat{v}_{k}(\tau) e^{-i \mathbf{k} \cdot \mathbf{x}} \\
= & \frac{1}{2} \int \frac{d^{3} k}{(2 \pi)^{3}}\left[\left(a_{-\mathbf{k}}+b_{-\mathbf{k}}\right) q_{k}(\tau)\right. \\
& \left.+\left(b_{\mathbf{k}}^{\dagger}+a_{\mathbf{k}}^{\dagger}\right) \bar{q}_{k}(\tau)\right] e^{-i \mathbf{k} \cdot \mathbf{x}},
\end{aligned}
$$

and

$$
\begin{aligned}
\hat{u}(\tau, \mathbf{x})= & \int \frac{d^{3} k}{(2 \pi)^{3}} \hat{u}_{k}(\tau) e^{-i \mathbf{k} \cdot \mathbf{x}} \\
= & \frac{1}{2 i} \int \frac{d^{3} k}{(2 \pi)^{3}}\left[\left(a_{-\mathbf{k}}-b_{-\mathbf{k}}\right) q_{k}(\tau)\right. \\
& \left.+\left(b_{\mathbf{k}}^{\dagger}-a_{\mathbf{k}}^{\dagger}\right) \bar{q}_{k}(\tau)\right] e^{-i \mathbf{k} \cdot \mathbf{x}} .
\end{aligned}
$$

In order to implement the perturbative analysis, we decompose the quadratic action (31) to the free and interaction parts. Correspondingly, the interaction Hamiltonians are obtained to be

$$
\begin{gathered}
H_{u u}^{\mathrm{int}}=-\frac{3}{2} I \epsilon \int \frac{d^{3} k}{(2 \pi)^{3}}\left(1-\frac{\mathbf{e}^{2}}{9 H^{2}}\left(\frac{\tau_{e}}{\tau}\right)^{4}\right) \frac{\hat{u}_{k}^{2}}{\tau^{2}} \\
H_{v v}^{\mathrm{int}}=-\frac{3}{2} I \int \frac{d^{3} k}{(2 \pi)^{3}}\left(\epsilon+4 \cos 2 \theta-\frac{\epsilon \mathbf{e}^{2}}{9 H^{2}}\left(\frac{\tau_{e}}{\tau}\right)^{4}\right) \frac{\hat{v}_{k}^{2}}{\tau^{2}} \\
H_{v D}^{\mathrm{int}}=2 \sqrt{6 I} \sin \theta \int \frac{d^{3} k}{(2 \pi)^{3}}\left(-\frac{2 \hat{D}_{k}}{\tau}+\hat{D}_{k}^{\prime}\right. \\
\left.+\frac{\mathbf{e}^{2}}{3 H^{2}}\left(\frac{\tau_{e}}{\tau}\right)^{4} \frac{\hat{D}_{k}}{\tau}\right) \frac{\hat{v}_{k}}{\tau} \\
H_{D D}^{\mathrm{int}}=\frac{1}{\epsilon} \int \frac{d^{3} k}{(2 \pi)^{3}} \frac{\mathbf{e}^{2}}{H^{2}}\left(\frac{\tau_{e}}{\tau}\right)^{4} \frac{\hat{D}_{k}^{2}}{\tau^{2}}
\end{gathered}
$$

It is important to note that the interactions (44), (45), and (46) become large after the time $\tau_{v}$ defined in (35) while from Eq. (37) we see that they remain small till the end of inflation. The interaction (47), however, becomes large after the time $\tau_{D}$ given in Eq. (34) and from Eq. (37) it is clear that it cannot be treated perturbatively for $\tau \in\left(\tau_{D}, \tau_{e}\right]$. Therefore, we should be careful about the time interval in which the interaction term (47) plays role in our subsequent analysis. It is worth mentioning that this interaction will not contribute to the pair production process and curvature perturbation power spectrum. It only contributes to the power spectrum of the isocurvature mode $D$.

Starting with the Bunch-Davies initial condition, the free mode functions are given by 


$$
v_{k}=u_{k}=D_{k}=\frac{i e^{-i k \tau}}{\sqrt{2 k^{3}} \tau}(1+i k \tau)
$$

Now, we have all we need in hand to calculate the number of produced pairs. Before doing this, it is useful to clarify the relation between the standard procedure of pair production by the Schwinger mechanism through computing the Bogoliubov coefficients and the perturbative analysis that we apply here. It is well known that a nontrivial background geometry, such as a time-dependent cosmological background, leads to a mixing between positive and negative frequency modes. In other words, the positive frequency mode function $q_{k}$ at asymptotic past $\tau \rightarrow-\infty$ is given by a linear combination of the positive and negative frequency modes at asymptotic future $\tau \rightarrow 0$. Choosing the vacuum $|\Omega\rangle$ in the asymptotic future by $\tilde{a}_{\mathbf{k}}|\Omega\rangle=\tilde{b}_{\mathbf{k}}|\Omega\rangle=0$, where

$\tilde{a}_{\mathbf{k}}=\alpha_{k} a_{\mathbf{k}}+\bar{\beta}_{k} b_{-\mathbf{k}}^{\dagger}, \quad \tilde{b}_{\mathbf{k}}=\bar{\beta}_{-k} a_{-\mathbf{k}}^{\dagger}+\alpha_{-k} b_{\mathbf{k}}$,

with $\alpha_{k}$ and $\beta_{k}$ being the Bogoliubov coefficients, and assuming the usual commutation relations between $\tilde{a}_{\mathbf{k}}$ and $\tilde{a}_{\mathbf{k}}^{\dagger}$, we have $\left|\alpha_{k}\right|^{2}-\left|\beta_{k}\right|^{2}=1$. By simple algebra one can express $a_{\mathbf{k}}$ and $b_{\mathbf{k}}$ in terms of $\tilde{a}_{\mathbf{k}}$ and $\tilde{b}_{\mathbf{k}}$ as follows

$$
a_{\mathbf{k}}=\bar{\alpha}_{k} \tilde{a}_{\mathbf{k}}-\bar{\beta}_{k} \tilde{b}_{-\mathbf{k}}^{\dagger}, \quad b_{\mathbf{k}}=\bar{\alpha}_{-k} \tilde{b}_{\mathbf{k}}-\bar{\beta}_{-k} \tilde{a}_{-\mathbf{k}}^{\dagger} .
$$

The number of the charged pairs produced from the infinite past to infinite future is

$$
\mathcal{N}_{k}=\left\langle\Omega\left|a_{\mathbf{k}}^{\dagger} a_{\mathbf{k}}\right| \Omega\right\rangle=\left\langle\Omega\left|b_{-\mathbf{k}}^{\dagger} b_{-\mathbf{k}}\right| \Omega\right\rangle=\left|\beta_{k}\right|^{2} .
$$

The associated Feynman diagrams giving nonzero contributions to Eq. (51) are shown in Fig. 2. In order to calculate $\mathcal{N}_{k}$ in Eq. (51), we can implement the in-in formalism instead of computing the Bogoliubov coefficient through the equation of motion (29). This is because the interaction terms are small during inflation, allowing for a perturbative in-in analysis.

The in-in formula for the correlation functions of a typical scalar mode $\delta X$ is [34,35]

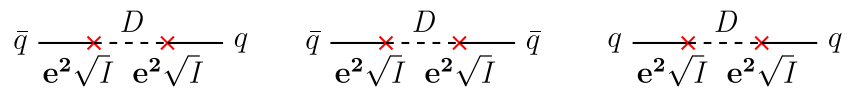

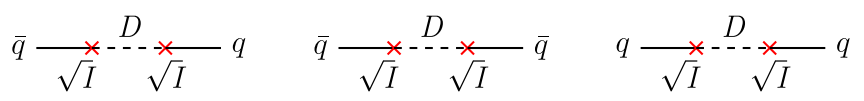

FIG. 2. Feynman diagrams corresponding to interaction Hamiltonian (46) giving rise perturbative pair production in (53). These transfer vertices show the interactions between the charged scalar field $q$ and its complex conjugate $\bar{q}$ with the gauge field excitation $D$.

$$
\begin{aligned}
\Delta\left\langle\delta X^{2}\left(\tau_{c}\right)\right\rangle= & \int_{\tau_{0}}^{\tau_{c}} d \tilde{\tau}_{1} \int_{\tau_{0}}^{\tau_{c}} d \tau_{1}\left\langle 0\left|H_{I}\left(\tilde{\tau}_{1}\right) \delta X^{2}\left(\tau_{c}\right) H_{I}\left(\tau_{1}\right)\right| 0\right\rangle \\
& -2 \operatorname{Re}\left[\int_{\tau_{0}}^{\tau_{c}} d \tau_{1} \int_{\tau_{0}}^{\tau_{1}} d \tau_{2}\right. \\
& \left.\times\left\langle 0\left|\delta X^{2}\left(\tau_{c}\right) H_{I}\left(\tau_{1}\right) H_{I}\left(\tau_{2}\right)\right| 0\right\rangle\right]+\cdots, \quad(52)
\end{aligned}
$$

in which $H_{I}$ are the interaction Hamiltonians in the interaction picture and $|0\rangle$ is the free vacuum defined in the absence of the interactions. Looking at (51), in order to find the number of pairs, one can easily calculate $\left\langle\Omega\left|a_{\mathbf{k}}^{\dagger} a_{\mathbf{k}^{\prime}}\right| \Omega\right\rangle$ by means of Eq. (52). The second term in the above formula vanishes since the effect of the creation operator on the free vacuum from the left vanishes and we simply have

$$
\begin{aligned}
\left\langle\Omega\left|a_{\mathbf{k}}^{\dagger} a_{\mathbf{k}^{\prime}}\right| \Omega\right\rangle & =\int_{-\infty}^{\tau_{e}} d \tilde{\tau}_{1} \int_{-\infty}^{\tau_{e}} d \tau_{1}\left\langle 0\left|H_{v D}^{\mathrm{int}}\left(\tilde{\tau}_{1}\right) a_{\mathbf{k}}^{\dagger} a_{\mathbf{k}^{\prime}} H_{v D}^{\mathrm{int}}\left(\tau_{1}\right)\right| 0\right\rangle \\
& \equiv \mathcal{N}_{k}(2 \pi)^{3} \delta^{3}\left(\mathbf{k}-\mathbf{k}^{\prime}\right),
\end{aligned}
$$

with

$\mathcal{N}_{k}=\left(\frac{3}{2}-\frac{4 \mathbf{e}^{2} M_{P}^{2} k^{4} \tau_{e}^{4}}{35 H^{2}}+\frac{8 \mathbf{e}^{4} M_{P}^{4} k^{8} \tau_{e}^{8}}{3675 H^{4}}\right) I \sin ^{2} \theta N_{k}^{2}$,

where $N_{k}=-\ln \left(-k \tau_{e}\right)$ is the number of e-folds counted from the time when the mode of interest $k$ has left the horizon till the time of end of inflation. Note that the integrals are taken from the past infinity to the end of inflation. This is because the interaction Hamiltonian $H_{v D}^{\text {int }}$ becomes nonperturbative only after $\tau_{v} \gtrsim \tau_{e}$ and therefore we are allowed to perform the integrals from the past infinity to the end of inflation.

The scale dependent of $\mathcal{N}_{k}$ is clear from the last two terms in bracket in Eq. (54). To quantify this more appropriately, let us define the scale $k_{D}$ for modes which leave the horizon at $\tau=\tau_{D}$, corresponding to $k_{D} \tau_{D}=-1$. Then using the expression for $\tau_{D}$ given in Eq. (34), we obtain

$\mathcal{N}_{k}=\left[\frac{3}{2}-\frac{4 \epsilon}{35}\left(\frac{k}{k_{D}}\right)^{4}+\frac{8 \epsilon^{2}}{3675}\left(\frac{k}{k_{D}}\right)^{8}\right] I \sin ^{2} \theta N_{k}^{2}$.

For observable CMB scales where $k \tau_{e} \sim k / k_{D} \rightarrow 0$, the dominant term in $\mathcal{N}_{k}$ is the first term in the bracket in Eq. (54). In next section, we relate this to the amplitude of quadrupolar statistical anisotropy. On the other hand, for modes which leave the horizon only toward the end of inflation corresponding to $k \gtrsim k_{D}, \mathcal{N}_{k}$ can start to grow. However, this period is short and inflation ends quickly afterward. In addition, these scales are exponentially small compared to observable scales and cannot have any interesting observables effects. In a sense, the possible 
nonperturbative pair creations for scales smaller than $k_{D}^{-1}$ becomes entangled with preheating mechanism of particle creations on small scales at the end of inflation.

In addition, we see that the number density of produced pairs is anisotropic, being proportional to $\sin ^{2} \theta$ where $\theta$ is the angle between mode number $\mathbf{k}$ and a preferred direction in the sky (the direction of anisotropy determined by the background electric field, which in our case is along the $x$-direction).

\section{CURVATURE AND ISOCURVATURE POWER SPECTRA}

In this section, we calculate the power spectra of the scalar modes in our model. We deal with a multiple field scenario with the scalar modes $u$ and $v$ coming from the quantum fluctuations of the complex inflaton field while the scalar mode $D$ coming from the quantum fluctuation of the electric field.

The comoving curvature perturbation is given by $\mathcal{R}=$ $-\psi+H \delta u$ where $\delta u$ is the velocity potential which is defined as $\delta T_{i}^{t}=\partial_{i} \delta u$. In the spatially flat gauge (21), the curvature perturbation reduces to $\mathcal{R}=H \delta u$ with

$$
\delta u=-\frac{q+\bar{q}}{2 \phi^{\prime}}=-\frac{v}{\phi^{\prime}} .
$$

The above result shows that only the real part of the quantum fluctuations of the inflaton contribute to the curvature perturbations, yielding

$$
\mathcal{R}=H \delta u=-\left(\frac{H}{\dot{\phi}}\right) \frac{v}{a} .
$$

The associated two-point correlation function is given by

$\left\langle\mathcal{R}_{\mathbf{k}}^{\dagger} \mathcal{R}_{\mathbf{k}^{\prime}}\right\rangle=\left(\frac{H}{\dot{\phi}}\right)^{2} \frac{\left\langle v^{\dagger} v\right\rangle}{a^{2}}=\frac{2 \pi^{2}}{k^{3}} \mathcal{P}_{\mathcal{R}}(2 \pi)^{3} \delta\left(\mathbf{k}-\mathbf{k}^{\prime}\right)$,

where $\mathcal{P}_{\mathcal{R}}$ is the dimensionless power spectrum of the curvature perturbations.

To find the power spectrum, first we need to find the twopoint correlation function for the real part of the scalar mode $v$ which is given by

$$
\left\langle v_{\mathbf{k}}^{\dagger} v_{\mathbf{k}^{\prime}}\right\rangle=\frac{H^{2}}{2 k^{3}}(2 \pi)^{3} \delta\left(\mathbf{k}-\mathbf{k}^{\prime}\right)+\Delta\left\langle v_{\mathbf{k}}^{\dagger} v_{\mathbf{k}^{\prime}}\right\rangle,
$$

where the first term is obtained using Eq. (48) coming from the free theory in the absence of interaction between the gauge field and the inflaton field. The second term in (59), $\Delta\left\langle v_{\mathbf{k}}^{\dagger} v_{\mathbf{k}^{\prime}}\right\rangle$, represents contributions from the interactions listed in Eqs. (44), (45), (46), and (47). The leading contributions in $\Delta\left\langle v_{\mathbf{k}}^{\dagger} v_{\mathbf{k}^{\prime}}\right\rangle$ come from the off-diagonal interaction Eq. (46) which are shown in the Feynman diagrams in Fig. 2, yielding

$$
\begin{aligned}
\Delta\left\langle v_{\mathbf{k}}^{\dagger} v_{\mathbf{k}^{\prime}}\right\rangle= & \frac{H^{2}}{k^{3}}\left(1-\frac{\mathbf{e}^{2}}{42 H^{2} N_{k}}+\frac{\mathbf{e}^{4}}{4312 H^{4} N_{k}^{2}}\right) \\
& \times 12 I \sin ^{2} \theta N_{k}^{2}(2 \pi)^{3} \delta^{3}\left(\mathbf{k}-\mathbf{k}^{\prime}\right) .
\end{aligned}
$$

Substituting Eq. (60) into Eqs. (59) and Eq. (58) we find the curvature perturbations power spectrum to be

$$
\mathcal{P}_{\mathcal{R}}=\frac{H^{2}}{8 \pi^{2} \epsilon M_{P}^{2}}\left(1+24 F(\beta) I \sin ^{2} \theta N_{k}^{2}\right),
$$

where we have defined the dimensionless parameter $\beta$ and the function $F(\beta)$ as follows

$$
\beta \equiv \frac{\mathbf{e}^{2} M_{P}^{2}}{42 H^{2} N_{k}}, \quad F(\beta) \equiv 1-\beta+\frac{9}{22} \beta^{2} .
$$

The above result coincides with the result obtained in [27] using a different gauge. Assuming $M_{P} / H \sim 10^{5}$ in chaotic inflation model, we would have $\beta \gtrsim 1$ for $\mathbf{e} \gtrsim 10^{-4}$. However, as shown in [27], in order to keep the anisotropies in tensor sector under perturbative control one actually requires $\mathbf{e} \lesssim 10^{-3}$, so in practice $\beta$ is not much bigger than unity.

Conventionally, the statistical anisotropies in curvature perturbation power spectrum can be parametrized in terms of quadrupole amplitude $g_{*}$, defined via

$$
\mathcal{P}_{\mathcal{R}}=\mathcal{P}_{\mathcal{R}}^{(0)}\left(1+g_{*} \cos ^{2} \theta\right),
$$

where $\mathcal{P}_{\mathcal{R}}^{(0)}$ is the isotropic power spectrum in the absence of gauge field. Comparing the above definition with our result obtained in Eq. (61), we have

$$
g_{*}=-24 I N_{k}^{2} F(\beta) .
$$

There are tight observational constraints on the amplitude of $g_{*}$, requiring $\left|g_{*}\right|<10^{-2}$ [28]. With $N_{k} \simeq 60$ for CMB scales, we conclude that $I \lesssim 10^{-7}$.

We can now relate the number density of the created pairs to the amplitude of quadrupole anisotropy $g_{*}$. Using Eqs. (55) and (64), and assuming $\beta \sim \mathcal{O}(1)$, we find $\mathcal{N}_{k} \sim g_{*}$. This is an interesting result, providing a direct link between the amplitude of quadrupole anisotropy and the number density of the created pairs. As discussed before, the conventional nonperturbative Schwinger mechanism will not take place during inflation and it is only the perturbative pair creation which operates during most of the period of inflation. However, the key difference in our model compared to previous works is that the complex scalar field is the inflaton field itself which at the same time is responsible for curvature perturbation. As a result, there is not much room for the efficiency of charged pair creation. This is unlike other hypothetical setups where the complex scalar field assumed to be a test 
field decoupled from the inflationary sector and also where the electric field was given as a background field with no dynamical mechanism for its generation.

To continue, we calculate the power spectra of the isocurvature modes which were not calculated in [27]. With the quadratic action (31) at hand we can easily calculate the power spectra of the other scalar modes as well. We note that the scalar mode $u$ is completely decoupled from the other modes and therefore it is an isocurvature mode. One can directly solve the full equation of motion for the mode $u$ from Eq. (31). However, the deviations from the standard free action are encoded in the interaction Hamiltonian (44) and it is easier to find the corrections through the in-in formula (52). The corrections in the power spectrum of $u$ come only from the interaction Hamiltonian (44) with the Feynman diagrams shown in the second row of Fig. 3. Performing the in-in integral, the correction in the power spectrum of $u$ is obtained to be

$\Delta\left\langle u_{\mathbf{k}}^{\dagger} u_{\mathbf{k}^{\prime}}\right\rangle=-\frac{H^{2}}{k^{3}}\left(1-\frac{\mathbf{e}^{2}}{84 H^{2} N_{e}}\right) \operatorname{IEN}_{k}(2 \pi)^{3} \delta^{3}\left(\mathbf{k}-\mathbf{k}^{\prime}\right)$.

Consequently, the corresponding dimensionless power spectrum is

$$
\mathcal{P}_{u}=\left(\frac{H}{2 \pi}\right)^{2}(1-(2-\beta)) I \epsilon N_{k} .
$$

What remains is the scalar mode $D$, the fluctuation of the gauge field. This mode couples to the curvature perturbation through the off-diagonal interaction (46). Therefore, it is an entropy mode. Following Ref. [36], the entropy mode interacts with the curvature perturbations so we should calculate their cross correlation. It is easy to see that the associated two-point function only receives contribution

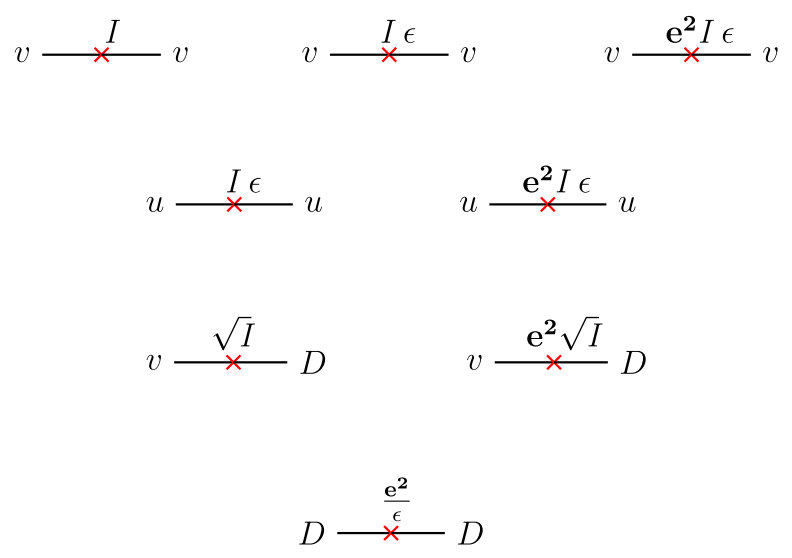

FIG. 3. The Feynman diagrams representing direct vertices originating from the interaction Hamiltonians listed in Eqs. (44), (45), (46), and (47). from the one-vertex Feynman diagrams between $v$ and $D$ which are shown in the third row of Fig. 3 . Correspondingly, the cross correlation between $v$ and $D$ modes is given by

$$
\begin{aligned}
& \Delta\left\langle v_{\mathbf{k}}^{\dagger} D_{\mathbf{k}^{\prime}}\right\rangle \\
& \quad=\frac{H^{2}}{k^{3}}\left(1-\frac{\mathbf{e}^{2}}{42 H^{2} N_{k}}\right) \sqrt{6 I} \sin \theta N_{k}(2 \pi)^{3} \delta^{3}\left(\mathbf{k}-\mathbf{k}^{\prime}\right),
\end{aligned}
$$

yielding the dimensionless correlation function

$$
\mathcal{P}_{v D}=2(1-\beta) \sqrt{6 I} \sin \theta N_{k} .
$$

Finally, we calculate the power spectrum of the mode $D$. For this purpose, we should be careful about the IR limit of the in-in integrals in Eq. (52) since the mode $D$ is perturbative only till the time $\tau=\tau_{D}$ given in Eq. (34). The free wave function is given by the Bunch-Davies vacuum (48) and is the same as the mode $v$ shown in Eq. (59). The corrections in the power spectrum of $D$ come from the last Feynman diagrams in Fig. 3, yielding

$$
\begin{aligned}
\Delta\left\langle D_{\mathbf{k}}^{\dagger} D_{\mathbf{k}^{\prime}}\right\rangle= & -\frac{H^{2}}{k^{3}}\left(1+\frac{\mathbf{e}^{2}}{4 H^{2} N_{D}}-\frac{3 \mathbf{e}^{4}}{1232 H^{4} N_{D}}\right) \\
& \times \frac{8}{7} I_{\sin ^{2} \theta N_{D}(2 \pi)^{3} \delta^{3}\left(\mathbf{k}-\mathbf{k}^{\prime}\right),}
\end{aligned}
$$

where $N_{D}=-\ln \left(-k \tau_{D}\right)$. The dimensionless power spectrum for the entropy mode $D$ then will be

$\mathcal{P}_{D}=\left(\frac{H}{2 \pi}\right)^{2}\left[1-\frac{16}{7}\left(1+\frac{21}{2} \beta-\frac{189}{44} \beta^{2} N_{D}\right) I \sin ^{2} \theta N_{D}\right]$.

We note that the contribution from the interaction Hamiltonian (47) becomes nonperturbative after the time $\tau_{D}$ while the mode $v$ is still perturbative. The duration between $\tau_{D}$ and $\tau_{e}$, however, cannot be too large since we need about 60 e-folds of inflation to solve the flatness and the horizon problems. Note that this difference originates from the induced mass term $\mathbf{e}^{2} A_{\mu} A^{\mu} \rho^{2}$. However, as argued before, this mass term should be negligible during most of the period of inflation to allow for slow-roll inflation. Consequently, the time difference between $\tau_{e}$ and $\tau_{D}$ is about 1-2 e-folds and we can practically set $N_{D} \simeq N_{k}$.

\section{SUMMARY AND DISCUSSIONS}

In this work, we studied the efficiency of the Schwinger pair production in a minimal setup of inflation in which the inflaton field is a complex scalar field charged under the $U(1)$ gauge field. We have shown that there are severe constraints on the efficiency of the Schwinger mechanism 
in this scenario. In our setup the nearly constant electric field and a quasi-de Sitter background are natural attractor solutions of the field equations, in contrast to the previous considerations where a constant and uniform electric field in a fixed dS background geometry has been imposed by hand. Due to the smallness of the electric field energy density during much of the period of inflation, charged pair production could only occur perturbatively in this setup. The standard Schwinger pair production can become efficient only when the induced mass term $\mathbf{e}^{2} A_{\mu} A^{\mu} \rho^{2}$ becomes significant. But in this limit a large effective mass is induced for the inflaton field which violates the slow-roll condition, ending inflation abruptly. Therefore, the nonperturbative Schwinger pair creation may take place only toward the final stages of inflation and on very small scales.

We have shown that the pair production in our inflationary model is negligible since the number of pairs in Eq. (54) turned out to be proportional to the anisotropic parameter $I$ which is tightly constrained by the CMB observations, $I \lesssim 10^{-7}$. More specifically, we have shown that the number of the created pairs is related to the amplitude of quadrupolar statistical anisotropy $g_{*}$ which is tightly constrained by cosmological observations. One may wonder if the extension of the setup to the case of isotropic model would yield significantly larger values of pair numbers. It is easy to see that this cannot be the case. The isotropic extension of our model was studied in [29] containing a triplet of $U(1)$ gauge field charged under complex scalar fields. There is no constraint from statistical anisotropy in the isotropic extension of the current model. However, the condition that the curvature perturbation power spectrum is nearly scale invariant requires that $I<10^{-4}$. Although this is about three orders of magnitude larger than the bound on $I$ in our anisotropic model, nonetheless the number of created pairs is small.

Recently it is shown that, in contrast to the Abelian U(1) case, when the Schwinger effect is driven by an SU(2) gauge field coupled to a charged scalar doublet, both the Schwinger pair production and the induced current decrease as the interaction strength increases [37,38]. It is argued that the isotropy of the $\mathrm{SU}(2)$ model plays a crucial role in suppression of the particle production rate and also the reduction of the induced current in the strong field limit.

It seems that if the inflaton field itself is a complex field (as in our model) and its quantum fluctuations to be responsible for the Schwinger pair production, then we cannot achieve significant number of pairs. One extension beyond our work is to apply the idea of quasisingle field inflation [35]. In this scenario the charged scalar field responsible for Schwinger pairs is a semiheavy charged scalar field while the inflaton field is a real scalar field coupled to the gauge field. This idea is a combination of $[21,23]$. Another option can be to look at the Schwinger mechanism in the model of charge hybrid inflation [39]. In this model the inflaton field is a real scalar field while the complex scalar field is the waterfall field which terminates inflation. The waterfall field is coupled to the $U(1)$ field which may lead to Schwinger pair production. In this setup, the sector responsible for generating curvature perturbation (the inflaton field) is different than the sector responsible for the pair creation (the waterfall field), so the CMB constraints may be relaxed and there may exist a corner of parameter space where the Schwinger mechanism may be efficient. We plan to come back to this question in the future.

Since the pair production in this minimal inflationary model is unmeasurably small on large (CMB) scales, therefore there is no need to consider the backreaction effects of these particles. Consequently, the Schwinger effect cannot have any large scale effects such as constraining inflationary magnetogenesis scenarios as envisaged in [7].

\section{ACKNOWLEDGMENTS}

We thank Jiro Soda for correspondences and comments on the draft and S. A. Hosseini Mansoori for assistance in XACT code. M. A. Gorji thanks the Yukawa Institute for Theoretical Physics at Kyoto University for hospitality during the "2019 YITP Asian-Pacific Winter School and Workshop on Gravitation and Cosmology" where this work was in its final stage.
[1] J. Martin, Lect. Notes Phys. 738, 193 (2008).

[2] L. Parker, Phys. Rev. Lett. 21, 562 (1968); Phys. Rev. 183, 1057 (1969); Phys. Rev. D 3, 346 (1971); 3, 2546(E) (1971).

[3] S. W. Hawking, Commun. Math. Phys. 43, 199 (1975); 46, 206(E) (1976).

[4] F. Sauter, Z. Phys. 69, 742 (1931); W. Heisenberg and H. Euler, Z. Phys. 98, 714 (1936); J. S. Schwinger, Phys. Rev.
82, 664 (1951); F. Gelis and N. Tanji, Prog. Part. Nucl. Phys. 87, 1 (2016).

[5] R. Sharma and S. Singh, Phys. Rev. D 96, 025012 (2017); A. Ferreiro, J. Navarro-Salas, and S. Pla, Phys. Rev. D 98, 045015 (2018).

[6] J. Garriga, Phys. Rev. D 49, 6327 (1994); 49, 6343 (1994); V. M. Villalba, Phys. Rev. D 52, 3742 (1995); M. B. Frb, 
J. Garriga, S. Kanno, M. Sasaki, J. Soda, T. Tanaka, and A. Vilenkin, J. Cosmol. Astropart. Phys. 04 (2014) 009.

[7] T. Kobayashi and N. Afshordi, J. High Energy Phys. 10 (2014) 166.

[8] W. Fischler, P. H. Nguyen, J. F. Pedraza, and W. Tangarife, Phys. Rev. D 91, 086015 (2015).

[9] I. I. Cotescu and C. Crucean, Phys. Rev. D 87, 044016 (2013); C. Crucean, Phys. Rev. D 85, 084036 (2012); C. Crucean and M. A. Bloi, Phys. Rev. D 93, 044070 (2016).

[10] A. Di Piazza, E. Lotstedt, A. I. Milstein, and C. H. Keitel, Phys. Rev. Lett. 103, 170403 (2009); G. V. Dunne, H. Gies, and R. Schutzhold, Phys. Rev. D 80, 111301 (2009); G. Torgrimsson, C. Schneider, J. Oertel, and R. Schtzhold, J. High Energy Phys. 06 (2017) 043; H. Taya, Phys. Rev. D 99, 056006 (2019); G. Torgrimsson, Phys. Rev. D 99, 096002 (2019).

[11] S. Weinberg, The Quantum Theory of Fields. Vol. 1: Foundations (Cambridge University Press, Cambridge, England, 2005).

[12] S. P. Kim and D. N. Page, Phys. Rev. D 78, 103517 (2008); C. Stahl, E. Strobel, and S. S. Xue, Phys. Rev. D 93, 025004 (2016).

[13] T. Hayashinaka, T. Fujita, and J. Yokoyama, J. Cosmol. Astropart. Phys. 07 (2016) 010; T. Hayashinaka and J. Yokoyama, J. Cosmol. Astropart. Phys. 07 (2016) 012; T. Hayashinaka and S. S. Xue, Phys. Rev. D 97, 105010 (2018).

[14] M. Banyeres, G. Domnech, and J. Garriga, J. Cosmol. Astropart. Phys. 10 (2018) 023.

[15] E. Bavarsad, C. Stahl, and S. S. Xue, Phys. Rev. D 94, 104011 (2016).

[16] R. Sharma, S. Jagannathan, T. R. Seshadri, and K. Subramanian, Phys. Rev. D 96, 083511 (2017); C. Stahl, Nucl. Phys. B939, 95 (2019); S. Chakraborty, S. Pal, and S. SenGupta, arXiv:1810.03478; T. Kobayashi and M.S. Sloth, arXiv:1903.02561.

[17] O. O. Sobol, E. V. Gorbar, M. Kamarpour, and S. I. Vilchinskii, Phys. Rev. D 98, 063534 (2018).

[18] W. Tangarife, K. Tobioka, L. Ubaldi, and T. Volansky, J. High Energy Phys. 02 (2018) 084; W. Tangarife, K. Tobioka, L. Ubaldi, and T. Volansky, arXiv:1706.00438.

[19] M. Giovannini, Phys. Rev. D 97, 061301 (2018).

[20] M. A. Watanabe, S. Kanno, and J. Soda, Phys. Rev. Lett. 102, 191302 (2009).
[21] J. J. Geng, B. F. Li, J. Soda, A. Wang, Q. Wu, and T. Zhu, J. Cosmol. Astropart. Phys. 02 (2018) 018.

[22] H. Kitamoto, Phys. Rev. D 98, 103512 (2018).

[23] W. Z. Chua, Q. Ding, Y. Wang, and S. Zhou, J. High Energy Phys. 04 (2019) 066.

[24] R. Emami, H. Firouzjahi, S. M. Sadegh Movahed, and M. Zarei, J. Cosmol. Astropart. Phys. 02 (2011) 005.

[25] R. Emami and H. Firouzjahi, J. Cosmol. Astropart. Phys. 10 (2013) 041.

[26] V. M. Villalba, Phys. Rev. D 60, 127501 (1999); V. M. Villalba and W. Greiner, Mod. Phys. Lett. A 17, 1883 (2002).

[27] X. Chen, R. Emami, H. Firouzjahi, and Y. Wang, J. Cosmol. Astropart. Phys. 08 (2014) 027.

[28] J. Kim and E. Komatsu, Phys. Rev. D 88, 101301 (2013); P. A. R. Ade et al. (Planck Collaboration), Astron. Astrophys. 594, A20 (2016); A. Durakovic, P. Hunt, S. Mukherjee, S. Sarkar, and T. Souradeep, J. Cosmol. Astropart. Phys. 02 (2018) 012.

[29] H. Firouzjahi, M. A. Gorji, H. Mansoori, A. Karami, and T. Rostami, arXiv:1812.07464.

[30] M. Peloso, L. Sorbo, and C. Unal, J. Cosmol. Astropart. Phys. 09 (2016) 001; A. Papageorgiou, M. Peloso, and C. Unal, J. Cosmol. Astropart. Phys. 09 (2018) 030.

[31] M. a. Watanabe, S. Kanno, and J. Soda, Prog. Theor. Phys. 123, 1041 (2010); N. Bartolo, S. Matarrese, M. Peloso, and A. Ricciardone, Phys. Rev. D 87, 023504 (2013).

[32] A. A. Abolhasani, R. Emami, J. T. Firouzjaee, and H. Firouzjahi, J. Cosmol. Astropart. Phys. 08 (2013) 016.

[33] K. D. Lozanov and M. A. Amin, J. Cosmol. Astropart. Phys. 06 (2016) 032.

[34] S. Weinberg, Phys. Rev. D 72, 043514 (2005).

[35] X. Chen and Y. Wang, J. Cosmol. Astropart. Phys. 04 (2010) 027.

[36] C. Gordon, D. Wands, B. A. Bassett, and R. Maartens, Phys. Rev. D 63, 023506 (2000).

[37] K. D. Lozanov, A. Maleknejad, and E. Komatsu, J. High Energy Phys. 02 (2019) 041.

[38] A. Maleknejad and E. Komatsu, arXiv:1808.09076.

[39] A. A. Abolhasani, R. Emami, and H. Firouzjahi, J. Cosmol. Astropart. Phys. 05 (2014) 016. 